\title{
OPEN Mapping wind erosion hazard with regression-based machine learning algorithms
}

\author{
Hamid Gholami ${ }^{1 凶}$, Aliakbar Mohammadifar ${ }^{1}$, Dieu Tien Bui ${ }^{2,3 凶}$ \& Adrian L. Collins ${ }^{4}$
}

Land susceptibility to wind erosion hazard in Isfahan province, Iran, was mapped by testing 16 advanced regression-based machine learning methods: Robust linear regression (RLR), Cforest, Non-convex penalized quantile regression (NCPQR), Neural network with feature extraction (NNFE), Monotone multi-layer perception neural network (MMLPNN), Ridge regression (RR), Boosting generalized linear model (BGLM), Negative binomial generalized linear model (NBGLM), Boosting generalized additive model (BGAM), Spline generalized additive model (SGAM), Spike and slab regression (SSR), Stochastic gradient boosting (SGB), support vector machine (SVM), Relevance vector machine (RVM) and the Cubist and Adaptive network-based fuzzy inference system (ANFIS). Thirteen factors controlling wind erosion were mapped, and multicollinearity among these factors was quantified using the tolerance coefficient (TC) and variance inflation factor (VIF). Model performance was assessed by RMSE, MAE, MBE, and a Taylor diagram using both training and validation datasets. The result showed that five models (MMLPNN, SGAM, Cforest, BGAM and SGB) are capable of delivering a high prediction accuracy for land susceptibility to wind erosion hazard. DEM, precipitation, and vegetation (NDVI) are the most critical factors controlling wind erosion in the study area. Overall, regression-based machine learning models are efficient techniques for mapping land susceptibility to wind erosion hazards.

Wind erosion, as an environmental problem, has many adverse effects on the economics of societies and the health of terrestrial and marine ecosystems ${ }^{1-3}$. Therefore, predicting land susceptibility to wind erosion hazards such as dust emissions from land surfaces is essential for mitigating its effects. Literature review shows that different tools and techniques have been proposed for investigating different aspects of wind erosion and its consequences, uniquely identifying regions prone to generating sediments for wind erosion, including remote sensing, data mining, and sediment fingerprinting ${ }^{4-7}$. However, these techniques require intensive field sampling with expensive laboratory analyses ${ }^{8}$, and as a result, they are not efficient for large spatial domains.

Recently, together with developments of geospatial technology and computer sciences, machine learning (ML) has received considerable attention with many successful applications in the spatial mapping of different environmental hazards such as land subsidence, gully erosion, landslides, and dust provenance, as well as mapping of soil properties (microbial dynamics, moisture, shear strength, soil taxa, bulk density, total nitrogen, organic carbon). However, to the best of our knowledge, exploration of the utility of advanced ML techniques in predicting land susceptibility to wind erosion has not been undertaken.

Typical ML models applied to date in different areas of environmental research include decision tree and linear equation models, the particle swarm optimization-adaptive network-based fuzzy inference system (PANFIS), genetic algorithms, support vector regression (SVR), artificial neural networks (ANN), hybrid models, random forest (RF), Wang and Mendel's (WM), partial least square regression (PLSR), principal component regression (PCR), Cubist, Bayesian additive regression trees (BART), radial basis function (RBF), extreme gradient boosting (XGBoost) and regression tree analysis ${ }^{8-15}$. Since, to date, a comprehensive study applying regression-based ML models to mapping wind erosion hazard has not been investigated, there remains a need for such work since wind erosion hazards are a major socio-economic challenge for some parts of the world. Accordingly, this work aimed to address this gap in the existing literature by providing a comprehensive assessing of the prediction performance of 16 regression-based ML models (robust linear regression (RLR), Cforest, non-convex penalized

\footnotetext{
${ }^{1}$ Department of Natural Resources Engineering, University of Hormozgan, Bandar-Abbas, Hormozgan, Iran. ${ }^{2}$ Institute of Research and Development, Duy Tan University, Da Nang 550000, Vietnam. ${ }^{3}$ GIS Group, Department of Business and IT, University of South-Eastern Norway, 3800 Bø i Telemark, Norway. ${ }^{4}$ Sustainable Agriculture Sciences, Rothamsted/Research, North Wyke, Okehampton EX20 2SB, Devon, UK. "email: hgholami@hormozgan.ac.ir; dieu.t.bui@usn.no
} 


\begin{tabular}{|l|l|l|}
\hline \multirow{2}{*}{ Effective factors } & \multicolumn{2}{l|}{$\begin{array}{l}\text { Collinearity } \\
\text { test }\end{array}$} \\
\cline { 2 - 3 } & TC & VIF \\
\hline AWC & 0.199 & 4.972 \\
\hline Bulk density & 0.169 & 5.93 \\
\hline Calcium carbonate percentage & 0.154 & 5.291 \\
\hline DEM & 0.183 & 5.46 \\
\hline EC & 0.109 & 5.61 \\
\hline ESP & 0.118 & 5.546 \\
\hline Land use & 0.855 & 1.169 \\
\hline Geology & 0.637 & 1.57 \\
\hline Precipitation & 0.315 & 3.177 \\
\hline Organic carbon content & 0.159 & 5.255 \\
\hline NDVI & 0.597 & 1.674 \\
\hline Soil texture & 0.203 & 4.932 \\
\hline Wind speed (m/s) & 0.656 & 1.523 \\
\hline
\end{tabular}

Table 1. Values of the TC and VIF for examining multicollinearity among the effective factors for wind erosion using the training dataset.

quantile regression (NCPQR), neural network with feature extraction (NNFE), monotone multi-layer perception neural network (MMLPNN), ridge regression (RR), boosting generalized linear model (BGLM), negative binomial generalized linear model (NBGLM), boosting generalized additive model (BGAM), spline generalized additive model (SGAM), spike and slab regression (SSR), stochastic gradient boosting (SGB), support vector machine (SVM), relevance vector machine (RVM), Cubist and adaptive network-based fuzzy inference system (ANFIS)) for mapping land susceptibility to the wind erosion hazard in the Isfahan province, central Iran. Using this case study, we provide more generic recommendations.

\section{Results}

Multicollinearity test. Table1 shows the values of the tolerance coefficient (TC) and the variance inflation factor (VIF) for the controlling factors for wind erosion. VIF $>10$ and $\mathrm{TC}<0.1$ indicate multicollinearity among the effective factors. Based on our results, the lowest TC value was obtained for electrical conductivity (EC), while the highest VIF value (5.93) value was calculated for bulk density. The results indicated the absence of any multicollinearity between the 13 factors controlling wind erosion in the study area.

Relative importance of the factors affecting wind erosion. The model with the highest performance (MMLPNN) was applied to quantify the relative importance of the effective factors for wind erosion. Based on Fig. 1, three factors, DEM (with relative importance 0.95), precipitation (with relative importance 0.8 ), and NDVI (with relative importance 0.54), were recognized as the most important factors controlling wind erosion in the study area. Wind erosion has been shown to be affected by many factors such as wind, precipitation, temperature, soil properties (texture, composition, and aggregation), topography, aerodynamic roughness, vegetation, and land use practice ${ }^{16}$.

\section{Discussion}

Maps of wind erosion hazard. The wind erosion hazard maps generated by 16 individual ML models are presented in Figs.2, 3, and 4. Table 2 indicates the area (percentage and $\mathrm{km}^{2}$ ) of the four land susceptibility classes (low, moderate, high, and very high) for wind erosion hazard estimated by the $16 \mathrm{ML}$ models. Based on the results of all 16 models, areas of land susceptibility to the low susceptibility class ranged between $15.5 \%$ (RVM and BGLM models) and 32.8\% (MMLPNN model). The minimum and maximum areas of moderate land susceptibility to wind erosion were estimated by the SGB $(0.6 \%)$ and SSR (15.7\%) models, respectively. The area of land categorized into the high susceptibility class ranged from 1.2\% (MMLPNN model) to 20.2\% (NCPQR model). Corresponding areas assigned to the very high class of land susceptibility to wind erosion hazard ranged from $41 \%$ (NBGLM model) to $65.2 \%$ (SGB).

Model performance assessment. Model performance for mapping wind erosion hazard was assessed by three indices (MAE, MBE, and RMSE; (Fig. 5)). Additionally, a Taylor diagram for both the training and evaluation datasets were constructed (Fig. 6). MMLPNN was selected as the most accurate model for mapping wind erosion hazard, while according to the RMSE and MAE, NBGLM was the weakest predictive model, and NCPQR was recognized as the overall worst model.

Based on all three statistical indicators of model performance and the Taylor diagram for the evaluation dataset, five models (MMLPNN, SGAM, Cforest, BGAM, and SGB) returned low errors. SSR and NBGLM had the lowest accuracies among the 16 models. Based on the Taylor diagram drawn for the training dataset, five models 


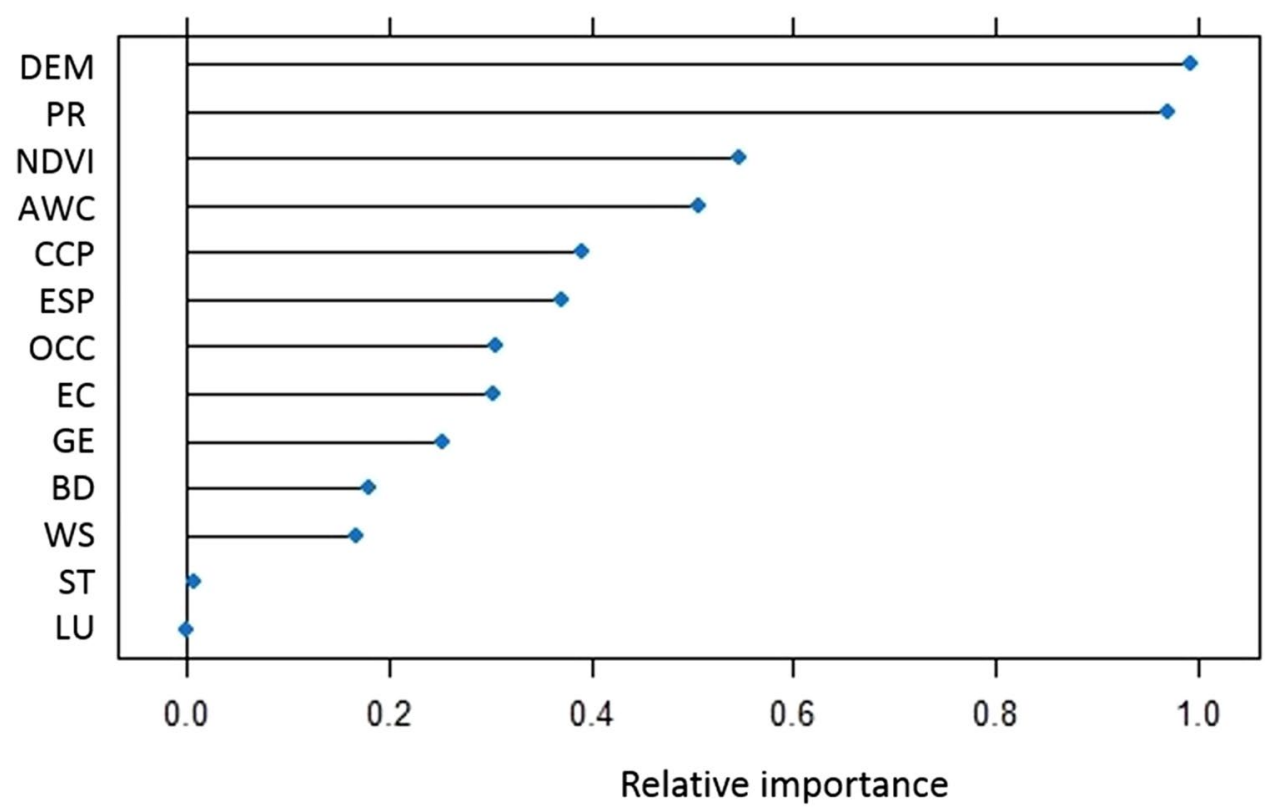

Figure 1. The relative importance of the effective factors for wind erosion estimated by MMLPNN. DEM, PR, NDVI, AWC, CCP, ESP, OCC, EC, GE, BD, WS, ST, and LU indicate digital elevation model, precipitation, normalized difference vegetation index, available water content, calcium carbonate content, exchangeable sodium percentage, organic carbon content, electrical conductivity, geology, bulk density, wind speed, soil texture, and land use, respectively.

(MMLPNN, Cforest, SGAM, SGB and NNFE) were identified as the most accurate predictive ML models for mapping wind erosion hazard in the study area, whereas NBGLM and RVM were the weakest predictive models.

Overall, MMLPNN, SGAM, Cforest, BGAM, and SGB were identified as the most accurate models for predicting land susceptibility to wind erosion. Based on MMLPNN (Fig. 2e), the four susceptibility classes covered $32.8 \%, 1.1 \%, 1.2 \%$ and $64.9 \%$ of the total area of Isfahan province, respectively. The land susceptibility map to wind erosion hazard generated using SGAM shows the high and very high susceptibility classes covered 5.4\% and $61.5 \%$ of the total area, respectively, whereas the low and moderate susceptibility classes occupied $27.4 \%$ and $5.6 \%$, respectively (Fig. 3d). According to Cforest (Fig. 2b), 26\%, 6.4\%, 6.6\%, and $61 \%$ of the total area belonged to the low, moderate, high and very high susceptibility classes, respectively. Using BGAM (Fig. 3c), the very high susceptibility class covered $62 \%$ of the study area, whereas the low, moderate, and high classes occupied $23.2 \%$, $7.8 \%$ and $7 \%$ of the total area, respectively. Finally, in the case of the SGB model (Fig. 3f), the results classified $32 \%, 0.6 \%, 2.2 \%$ and $65.2 \%$ of the study area as low, moderate, high, and very high susceptibility, respectively.

The map of wind erosion hazard produced by MMLPNN is the most accurate. Overall, multi-layer perception networks (MLPS) as universal estimators are well-known techniques for system identification. The monotonicity of MMLPNN does not depend on the quality of the training data because it is guaranteed by its structure ${ }^{17}$. GAM with spline function (SGAM) was one of the 5 most accurate models for wind erosion hazard mapping. The spline functions allow the flexible representation of non-linear marginal relationships of the explanatory and response variables without the necessity to define a specific function ${ }^{18}$. Cforest, as a random forest (RF) model, uses conditional inference trees for prediction ${ }^{19}$. Several studies confirm the performance of RF as a suitable model for spatial predictions of environmental hazards. For example ${ }^{20}$, reported that the RF model is the best model for digital mapping of soil carbon fractions.

Some studies ${ }^{21}$ have also argued that RF has the highest predictive capability for modelling landslide susceptibility in comparison with other ML models. Some previous studies ${ }^{22}$ have also reported that in comparison with other methods, RF has better performance in estimating $\mathrm{PM}_{2.5}$ monthly concentration. In this study, we applied the boosting with generalized additive model (BGAM), and based on the indicators for examining model performance, this model exhibited satisfactory performance and was selected as one of the five most accurate models for mapping wind erosion hazard. Boosting is a technique for improving prediction rules, and it can be applied to classification and regression methods to increase the accuracy of the predictions ${ }^{23}$. SGB is related to both boosting and bagging ${ }^{24,25}$. Previous research ${ }^{26}$ has reported that SGB provides stable predictions for tree species presence.

\section{Conclusions}

This research assessed the performance of 16 individual regression-based ML algorithms for mapping land susceptibility to wind erosion hazard in an arid region in central Iran. In all, 13 effective factors for wind erosion were considered and regions with active wind erosion were mapped using a "wind erosion inventory map". Based on three statistical indicators and a Taylor diagram, the MMLPNN model was the most accurate model. 


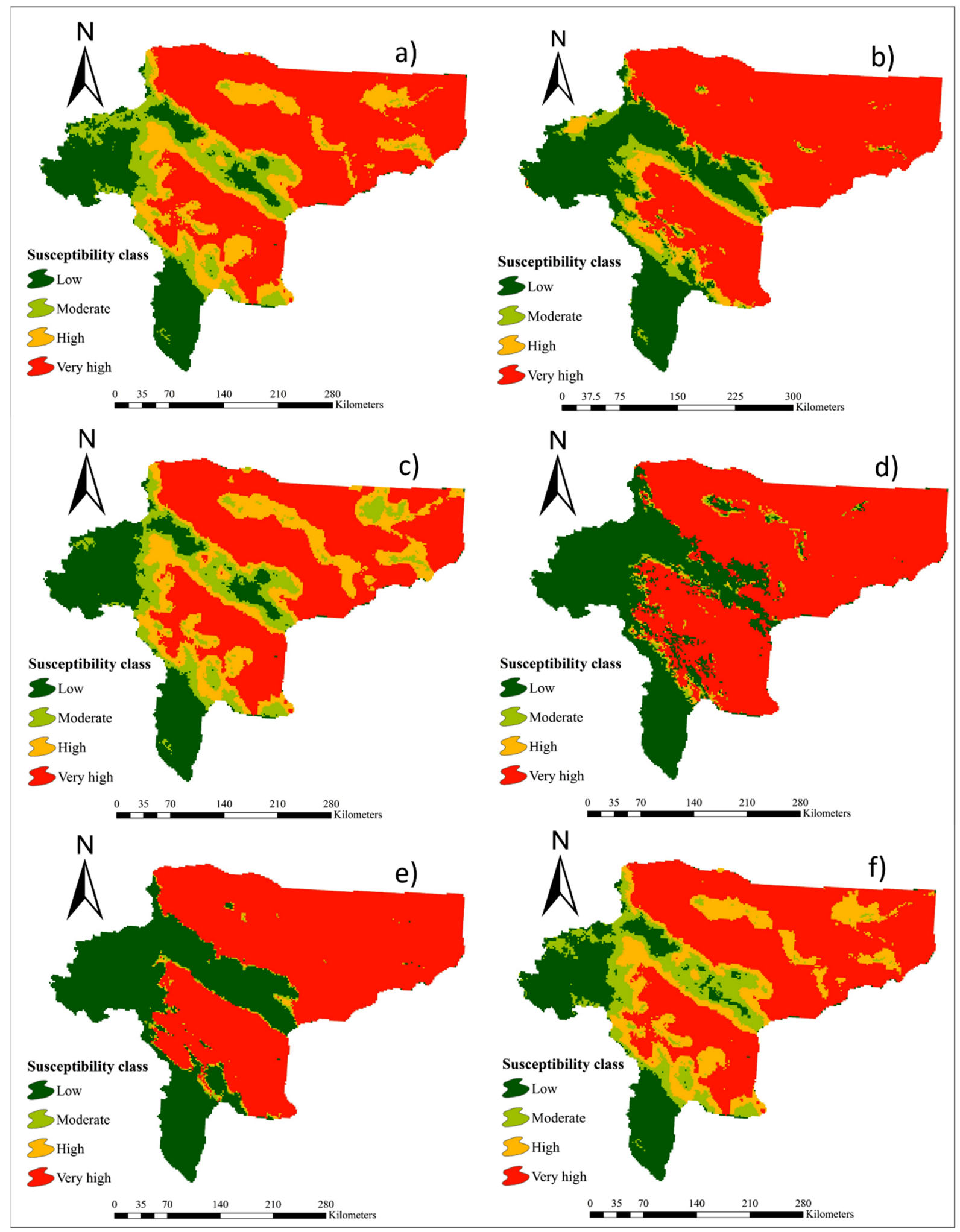

Figure 2. Maps of wind erosion hazard generated by: (a) RLR, (b) Cforest, (c) NCPQR, (d) NNFE, (e) MMLPNN, and (f) RR. The values for pixels was estimated by R software (https://CRAN.R-project.org/doc/ FAQ/R-FAQ.html) and then, values of pixels were mapped by ArcGIS 10.4.1 (https://www.esri.com/en-us/about /about-esri/overview). 


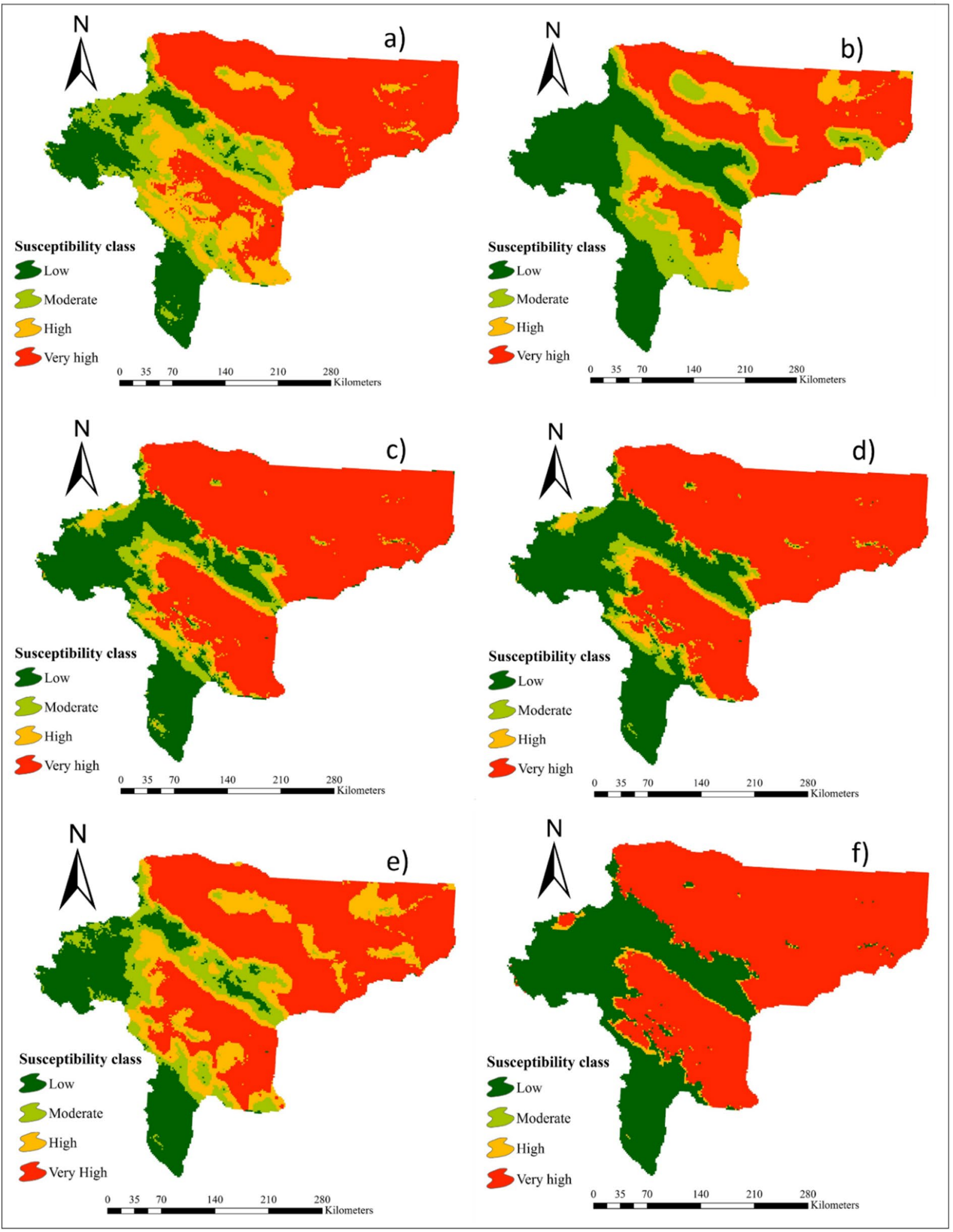

Figure 3. Maps of wind erosion hazard generated by: (a) BGLM, (b) NBGLM, (c) BGAM, (d) SGAM, (e) SSR, and (f) SGB. The values for pixels was estimated by R software (https://CRAN.R-project.org/doc/FAQ/R-FAQ. html) and then, values of pixels were mapped by ArcGIS 10.4.1 (https://www.esri.com/en-us/about/about-esri/ overview). 


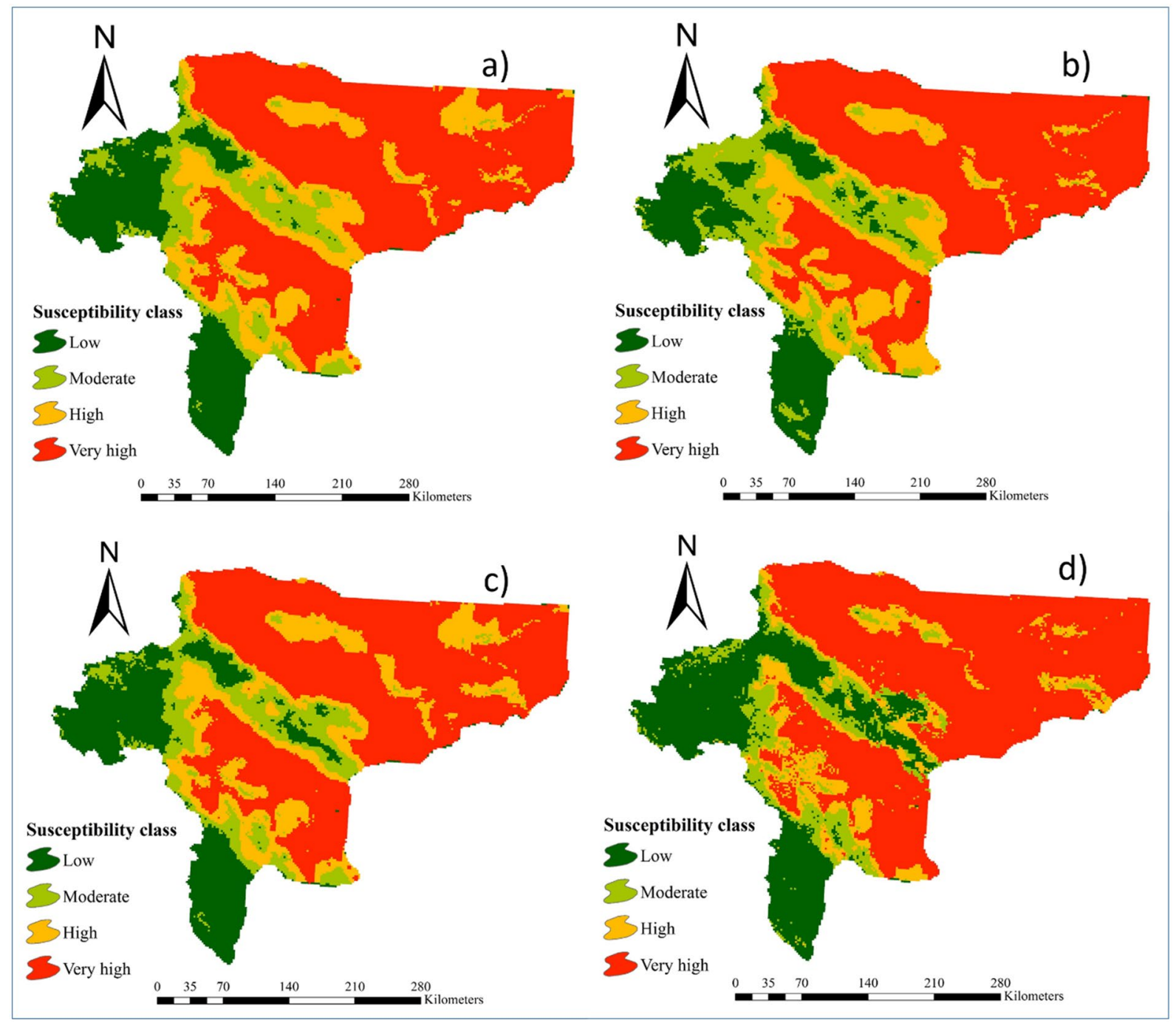

Figure 4. Maps of wind erosion hazard generated by: (a) SVM, (b) RVM, (c) Cubist and (d) ANFIS. The values for pixels was estimated by R software (https://CRAN.R-project.org/doc/FAQ/R-FAQ.html) and then, values of pixels were mapped by ArcGIS 10.4.1 (https://www.esri.com/en-us/about/about-esri/overview).

We conclude that MMLPNN is powerful tool for mapping wind erosion hazard in arid and semi-arid region ecosystems worldwide. We recommend that future work should focus on testing and comparing the performance of regression-based and classification-based ML models for the mapping and spatial modelling of wind erosion and dust sources to ensure that robust evidence is provided to support management decisions.

\section{Material and methods}

Study area. Isfahan province (Fig. 7), an arid region, is located in central Iran, between the latitudes $30^{\circ} 45^{\prime} 59.51^{\prime \prime}$ to $34^{\circ} 27^{\prime} 13.27^{\prime \prime} \mathrm{N}$, and between the longitudes $49^{\circ} 41^{\prime} 53.86^{\prime \prime}$ to $55^{\circ} 30^{\prime} 13.67^{\prime \prime} \mathrm{E}$. It is experiencing intensive wind erosion on the southeastern side (Segzi plain) and its northern parts. Based on a digital elevation model (DEM), there is high variability in altitude with maximum and minimum elevations ranging between $686 \mathrm{~m}$ (in the northern part of the study area and southern parts of Dasht-e-Kavir) to $4398 \mathrm{~m}$ (in the vicinity of the Dena Mountain in the southwestern part of the study area). The average annual precipitation ranges between $72 \mathrm{~mm}$ (in the eastern part with a corresponding annual mean temperature of $18^{\circ} \mathrm{C}$ ) and $320 \mathrm{~mm}$ (in the western part with an average annual temperature of $13^{\circ} \mathrm{C}$ ).

Factors controlling wind erosion. Different environmental and climatic factors are controlling wind erosion phenomena in drylands. Environmental variables affecting wind erosion include soil properties, lithology, land use, vegetation cover, topography, and elevation ${ }^{1,8,27}$. Previous research ${ }^{28}$ introduced a local wind erosion climatic index based on the wind speed and effective precipitation index developed by ${ }^{29}$ for applying in the Chepil wind erosion equation (WEQ). 


\begin{tabular}{|c|c|c|c|c|c|c|c|c|}
\hline \multirow[b]{3}{*}{ Model } & \multicolumn{8}{|c|}{ Susceptibility class } \\
\hline & \multicolumn{2}{|c|}{ Low } & \multicolumn{2}{|l|}{ Moderate } & \multicolumn{2}{|l|}{ High } & \multicolumn{2}{|l|}{ Very high } \\
\hline & Area $\left(\mathrm{km}^{2}\right)$ & Area (\%) & Area $\left(\mathrm{km}^{2}\right)$ & Area (\%) & Area $\left(\mathbf{k m}^{2}\right)$ & Area (\%) & Area $\left(\mathrm{km}^{2}\right)$ & Area (\%) \\
\hline RLR & 18,870 & 17.7 & 13,168 & 12.3 & 18,742 & 17.5 & 55,965 & 52.5 \\
\hline Cforest & 27,759 & 26 & 7101 & 6.4 & 7084 & 6.6 & 64,779 & 61 \\
\hline NCPQR & 21,076 & 19.7 & 12,963 & 12.1 & 21,554 & 20.2 & 51,174 & 48 \\
\hline NNFE & 33,748 & 31.6 & 1717 & 1.6 & 1894 & 1.8 & 69,429 & 65 \\
\hline MMLPNN & 35,007 & 32.8 & 1195 & 1.1 & 1296 & 1.2 & 69,295 & 64.9 \\
\hline $\mathrm{RR}$ & 18,857 & 17.7 & 12,945 & 12.1 & 19,470 & 18.2 & 55,454 & 52 \\
\hline BGLM & 16,356 & 15.4 & 15,126 & 14.2 & 20,484 & 19.2 & 54,474 & 51.2 \\
\hline NBGLM & 32,702 & 30.5 & 13,433 & 12.6 & 16,992 & 15.9 & 43,646 & 41 \\
\hline BGAM & 24,767 & 23.2 & 8295 & 7.8 & 7545 & 7 & 66,147 & 62 \\
\hline SGAM & 29,148 & 27.4 & 5957 & 5.6 & 5752 & 5.4 & 65,627 & 61.6 \\
\hline SSR & 18,894 & 17.7 & 16,719 & 15.7 & 23,510 & 22 & 47,562 & 44.6 \\
\hline SGB & 34,541 & 32 & 60 & 0.6 & 2310 & 2.2 & 69,844 & 65.2 \\
\hline SVM & 18,214 & 17 & 11,382 & 10.7 & 20,374 & 19 & 56,783 & 53.3 \\
\hline RVM & 16,364 & 15.4 & 15,767 & 14.8 & 18,523 & 17.3 & 56,098 & 52.5 \\
\hline Cubist & 19,326 & 18.2 & 12,543 & 11.7 & 19,797 & 18.5 & 55,077 & 51.6 \\
\hline ANFIS & 24,022 & 22.6 & 10,391 & 9.8 & 12,873 & 12 & 59,167 & 55.6 \\
\hline
\end{tabular}

Table 2. Land susceptibility classes to wind erosion hazard calculated by 16 individual ML models.

Soil characteristics. Seven soil characteristics (e.g., available water content (AWC) (Fig. 8a), bulk density (Fig. 8b), calcium carbonate percentage (Fig. 8c), electrical conductivity (EC) (Fig. 8d), exchangeable sodium percentage (ESP)(Fig. 8e), organic carbon content (OCC)(Fig. 8f) and soil texture (Fig. 9a)) were extracted from the world soil map ${ }^{30}$ and mapped by interpolation in ArcGIS 10.4.1. It should be noted that a total of 803 points (Fig. 7) were used for generating spatial maps.

Lithology and land use. Lithology (Fig. 9b) and land use (Fig. 9c) were mapped spatially based on the maps produced by the Forests, Rangelands, and Watershed Management Organization of Iran (FRWMOI).

Vegetation cover. The normalized difference vegetation index (NDVI) (Fig. 9d) ${ }^{31}$ as the most common index used for the spatial mapping of vegetation cover was applied in our study. NDVI is the difference between the red (RED) and near-infrared (NIR) band combination divided by the sum of the red and near-infrared band combination (Eq. 1).

$$
\mathrm{NDVI}=\left(\mathrm{NIR}_{\mathrm{b} 4}-\mathrm{RED}_{\mathrm{b} 3}\right) /\left(\mathrm{NIR}_{\mathrm{b} 4}+\mathrm{RED}_{\mathrm{b} 3}\right)
$$

Elevation. A digital elevation model (DEM) (Fig. 9e) for the study area was generated using shuttle radar topography mission (SRTM) images with a $30^{\star} 30 \mathrm{~m}$ resolution ${ }^{8}$.

Climatic variables. Wind speed (Fig. 9f) and precipitation (Fig. 10a) were used as climatic factors affecting wind erosion. The spatial maps of these variables were generated based on the daily average wind speed and total annual precipitation data from 23 meteorological stations located in the Isfahan province. All spatial maps of factors controlling wind erosion were generated in ArcGIS 10.4.1.

Inventory map of wind erosion. An inventory map shows regions with active three-stage processes, comprising detachment, transportation, and sedimentation due to wind erosion. An inventory map is needed for predicting land susceptibility to wind erosion hazard. We used a map of regions with active wind erosion produced by the Forest, Rangeland and Watershed Management Organization of Iran (FRWMOI) (Fig. 10b). Based on the inventory map, wind erosion active regions covered $\sim 10,961 \mathrm{~km}^{2}$ (440 pixels) in the study area. Pixels with active wind erosion were randomly selected for the training (70\% or 308 pixels) and validation (30\% or 112 pixels) datasets for the ML models (Fig. 10c). Based on field work and FRWMOI, inventory map of wind erosion was generated in ArcGIS 10.4.1.

Multicollinearity among the factors controlling wind erosion. The tolerance coefficient (TC) (Eq. 2) and variance inflation factor (VIF) (Eq. 3) 8,15,32 were applied to examine multicollinearity among the factors for wind erosion in the Isfahan province.

$$
\mathrm{TC}=1-\mathrm{R}^{2}
$$



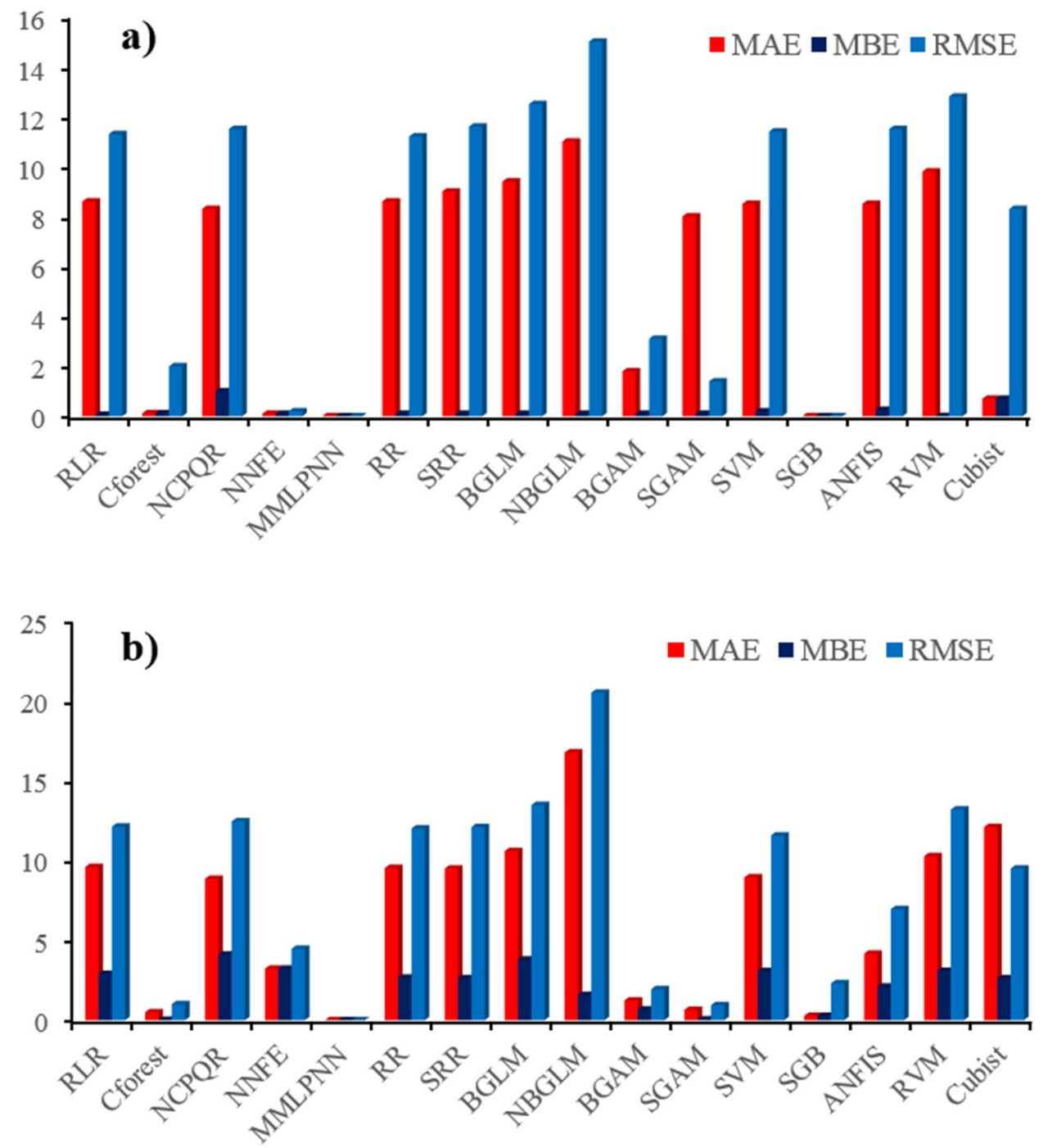

Figure 5. The values of the statistical indicators were used to evaluate model performance; (a) training dataset and (b) evaluation dataset.

$$
\mathrm{VIF}=\left[\frac{1}{\mathrm{TC}}\right]
$$

where $\mathrm{R}^{2}$ is the regression coefficient. If the TC is $<0.1$ and the VIF is $>10$, both coefficients signify a multicollinearity problem.

Background of the ML algorithms used. This section briefly describes the 16 individual regressionbased ML algorithms, which were adopted for mapping wind erosion hazard. These algorithms are available in the caret package, in R software.

Robust linear regression (RLR). Robust regression is designed to overcome some limitations of traditional parametric and non-parametric methods. Available robust regression methods include M-estimates ${ }^{33}$, R-estimates ${ }^{34}$, least median of squares (LMS) estimates ${ }^{35}$, least trimmed squares (LTS) estimates and S-estimates $^{36}$, generalized S-estimates (GS-estimates) ${ }^{37}$ and MM-estimates ${ }^{38}$. We used a robust linear regression model with M-estimates for predicting land susceptibility to wind erosion.

Cforest. Random forest (RF), introduced by ${ }^{39}$, is the most popular method for regression and classification in decision tree learning ${ }^{40}$. RF makes a large number of decision trees in the training phase, and then by averaging the output values of the trees, the output of the model is finalized. Cforest is a type of RF commonly applied for prediction purposes ${ }^{19}$. 

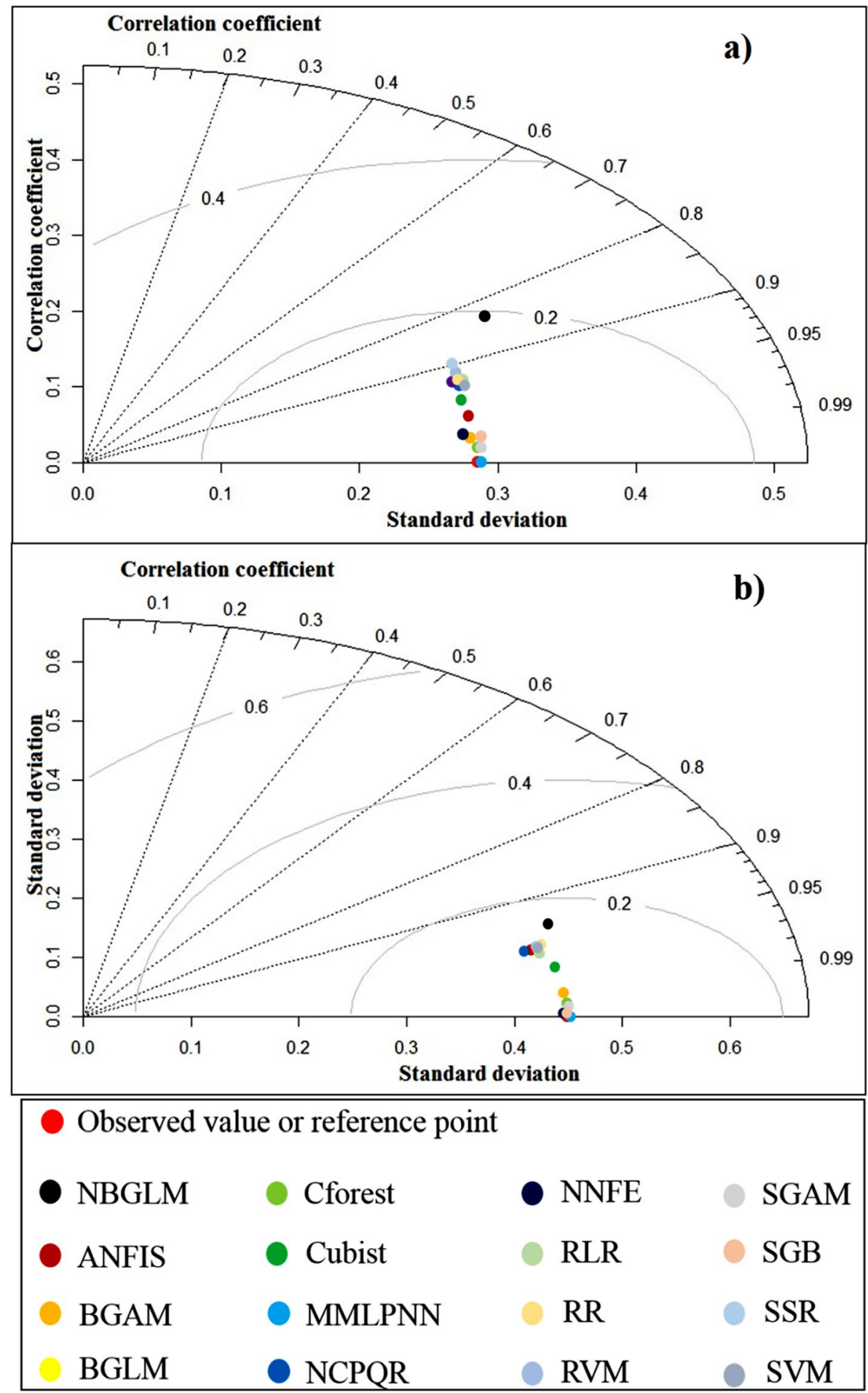

Figure 6. Taylor diagrams for assessing the performance of the models in this research; (a) training dataset, and (b) evaluation dataset.

Non-convex penalized quantile regression (NCPQR). Quantile regression (QR) has gained considerable attention in different fields of modelling since the work of ${ }^{41}$. In comparison with mean regression (MR), QR provides an alternative that is more efficient when the error term follows a non-normal heavy-tailed distribution ${ }^{42}$. We used a penalized QR with a non-convex function ${ }^{42}$ for mapping wind erosion hazard.

Neural networks (NN). NN can accurately approximate complicated non-linear input/output relationships ${ }^{43}$. The NN structure includes a set of interconnected units or neurons that estimates the non- 


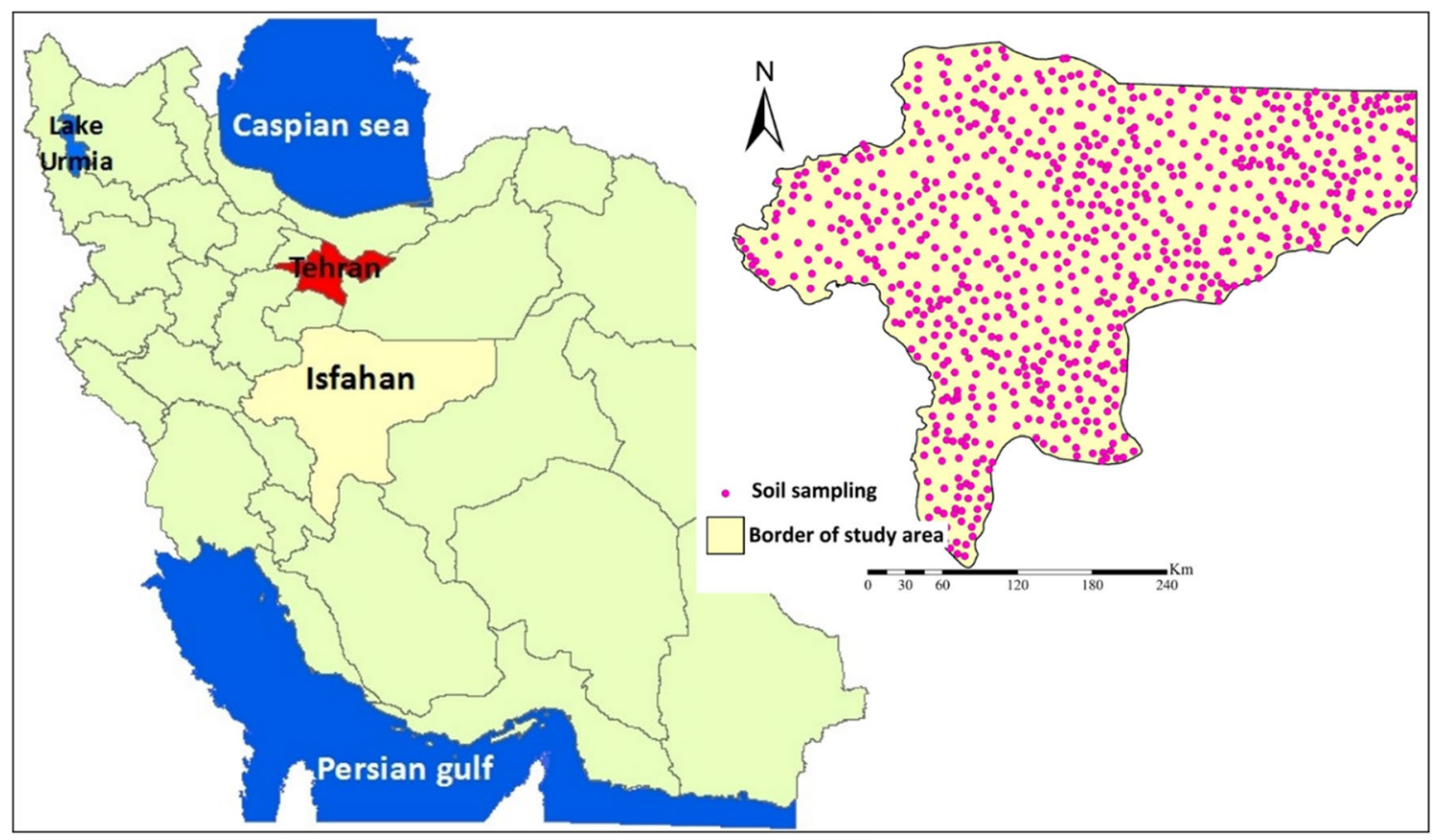

Figure 7. Location of the study area in Iran and sampling sites used for this study. Soil sampling sites were extracted from the world soil map ${ }^{30}$ and then, these sites were mapped in ArcGIS 10.4.1 (https://www.esri.com/ en-us/about/about-esri/overview).

linear correlations between each variable. The input neurons or predictor variables are connected to a single or multiple layers of hidden neurons, which are then linked to the output neurons ${ }^{44}$. We used a NN with the feature extraction algorithm (NNFE) ${ }^{45}$ and a monotone multi-layer perception neural network (MMLPNN) ${ }^{46}$ for mapping wind erosion hazard. The feature extractors used textural features based on the spatial relationships between pixels ${ }^{45}$.

Ridge regression with variable selection. Ridge regression (RR), which was proposed by ${ }^{47}$, is expressed as follows (Eq. 4):

$$
L(w)=\sum_{i=1}^{n}\left(y_{i}-\tilde{y}_{i}\right)^{2}=\sum_{i=1}^{n}\left(y_{i}-w \cdot x_{i}\right)^{2}
$$

Given a set of $n$ vectors, $x_{1}, \ldots, x_{n}$ in $R^{m}$, where $m$ is the number of properties, and the dependent variable $\mathrm{y}_{\mathrm{i}} \in R, i=1, \ldots, n$, the objective is to minimize the loss function, i.e., the discrepancy between the real values $\mathrm{y}_{\mathrm{i}}$ and the predicted values $\tilde{y}_{i}=w . x$.

We applied a RR model with a kernel function ${ }^{48}$ as follows:

$$
\tilde{y}=f(x)=\sum_{i=1}^{n} \beta_{i} K\left(x, x_{i}\right)
$$

where $K\left(x, x_{i}\right)$ is the kernel function and $\beta_{i}$ is the weighting.

Generalized linear models (GLMs). GLMs have been applied to a wide range of research ${ }^{49}$. GLMs have three components, comprising an observation model, a linear predictor, and an invertible link function ${ }^{50}$. Using boosting with GLMs can improve prediction accuracy ${ }^{23}$. We applied two GLMs; boosting GLM (BGLM) and negative binomial GLM (NBGLM) ${ }^{51}$.

Generalized additive models (GAMs). $\quad \mathrm{GAMs}^{52}$ can be expressed as follows:

$$
e\left(\mu_{i}\right)=Z_{i}^{*} \cdot \beta+\sum_{j} f_{j}\left(x_{i j}\right)
$$

with

$$
\mu_{i}=E\left(Y_{i}\right), \text { and } Y_{i} \sim E F\left(\mu_{i}, \varnothing\right),
$$

where $Y_{i}$ is the $i$ th value of the response variable from an exponential distribution family (EF) with a location parameter $\left(\mu_{i}\right)$ and a scale parameter $(\varnothing), Z_{i}^{*}$ indicates the $i$ th row of a parametric model matrix with the vector 


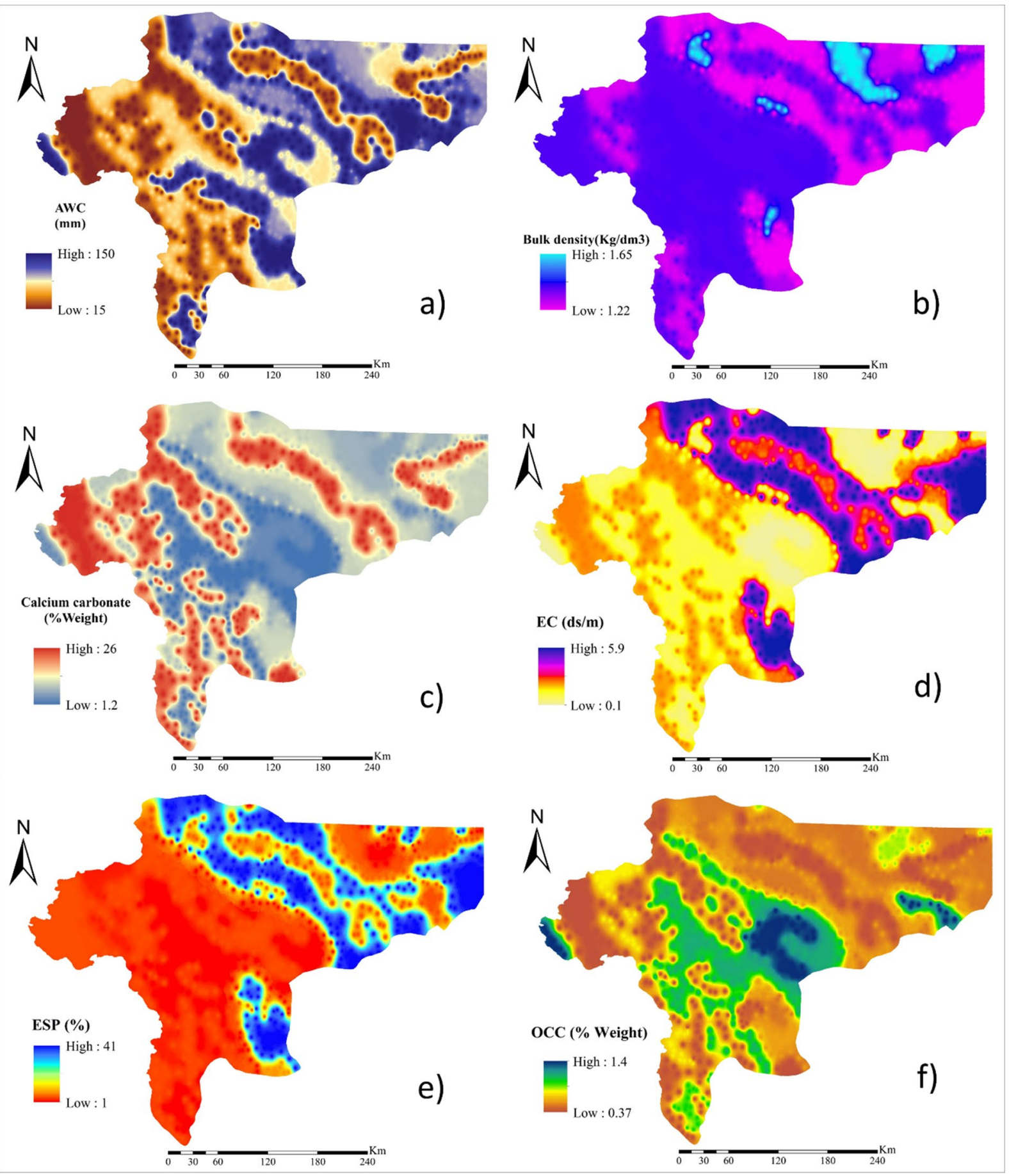

Figure 8. Spatial maps of soil characteristics: (a) AWC; (b) bulk density; (c) calcium carbonate percentage; (d) EC; (e) ESP, and; (f) OCC. All these factors were mapped spatially in ArcGIS 10.4.1 (https://www.esri.com/ en-us/about/about-esri/overview).

$\beta, f_{j}$ shows unknown functions and $x_{i j}$ indicates the $i$ th value of the $j$ th variable. $g\left(\mu_{i}\right)$ is the link function. We applied two GAMs, comprising boosting (BGAM) and spline (SGAM) ${ }^{18}$.

Spike and slab regression (SSR). SSR is one of the typical variable selection approaches in regression settings, and this model has been applied widely in challenging problems ${ }^{53}$. SSR was proposed by ${ }^{54}$ and can be expressed as follows ${ }^{53}$ :

$$
y_{i}=\beta_{1,0} x_{i, 1}+\cdots+\beta_{p, 0} x_{i, p}+\varepsilon_{i}, i=1, \ldots, n,
$$




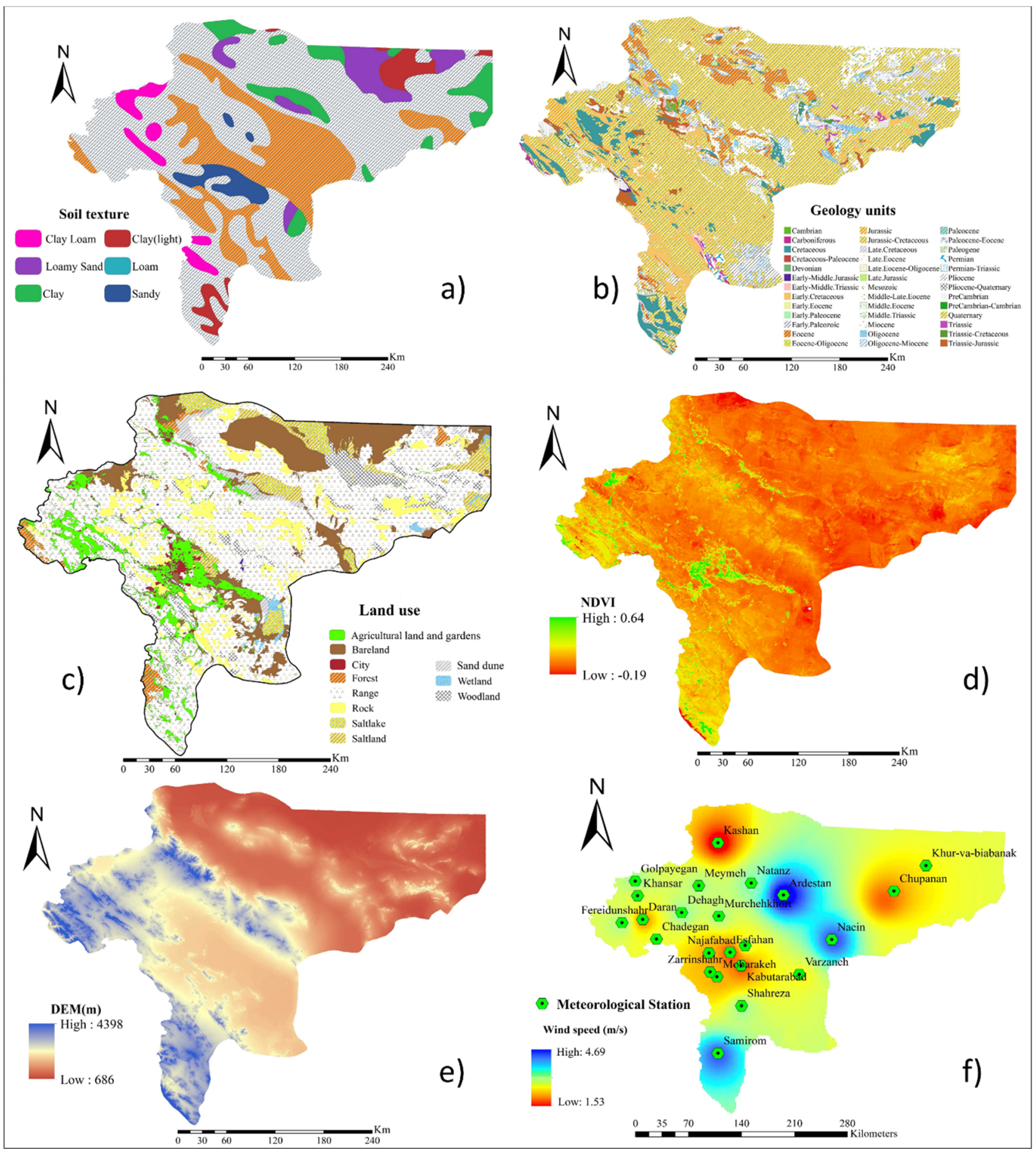

Figure 9. Spatial maps of: (a) soil texture; (b) geology; (c) land use; (d) NDVI; (e) DEM, and; (f) wind speed. All these factors were mapped spatially in ArcGIS 10.4.1 (https://www.esri.com/en-us/about/about-esri/overv iew).

where $\left(\varepsilon_{\mathrm{i}}\right)_{1 \leq \mathrm{i} \leq \mathrm{n}}$ are independent random variables such as $\mathrm{E}\left(\varepsilon_{\mathrm{i}}\right)=0$ and $\mathrm{E}\left(\varepsilon_{i}^{2}\right)=\sigma_{0}^{2}>0$. Write $\mathrm{X}$ for the $\mathrm{n} \times \mathrm{p}$ design matrix corresponding to $(1)$ and $\beta_{0}=\left(\beta_{0,1}, \ldots, \beta_{0, P}\right)^{T}$ for the true regression parameter. The variables $x_{i}=\left(x_{i, 1}, \ldots, x_{i, p}\right)^{T}$ and the response-vector $y=\left(y_{1}, \ldots, y_{n}\right)^{T}$ are assumed to the standardized such that: $\sum_{i=1}^{n} x_{i, k}=0, \sum_{i=1}^{n} x_{i, k}^{2}=n, \sum_{i=1}^{n} y_{i}=0$.

Stochastic gradient boosting (SGB). SGB or gradient boosting machine, proposed by ${ }^{24}$ is a hybrid algorithm that combines both the advantages of bagging and boosting. This model makes additive regression models by the least-squares at each iteration. 


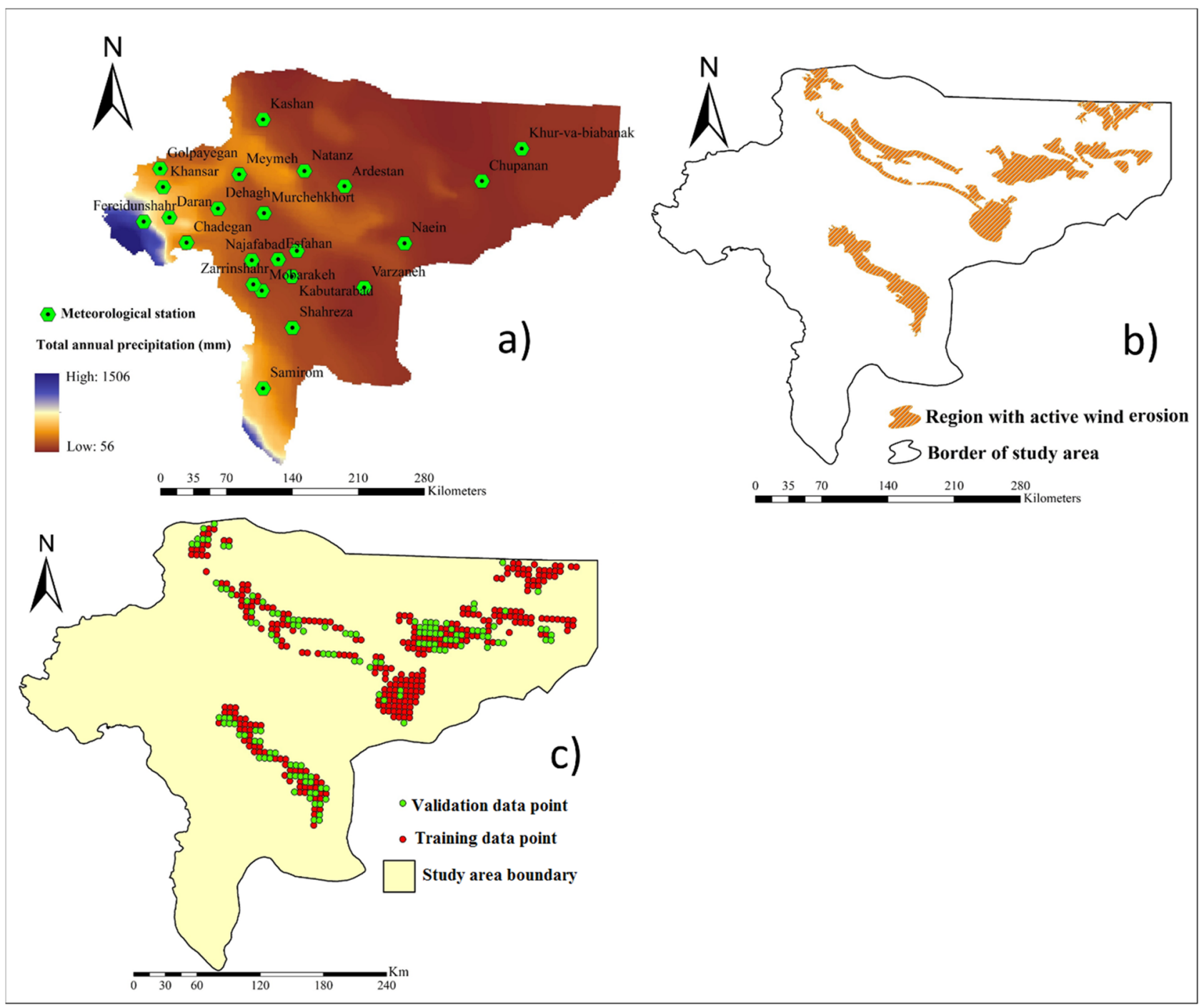

Figure 10. Spatial maps of: (a) total annual precipitation; (b) locations of the pixels with active wind erosion, and; (c) locations of the training and validation data points. All these characteristics were mapped spatially in ArcGIS 10.4.1 (https://www.esri.com/en-us/about/about-esri/overview).

Support and relevance vector machine (SVM and RVM) algorithms. The relevance vector machine (RVM) is a probabilistic sparse kernel model identical in functional form to the support vector machine (SVM). SVM is a very successful approach to supervised learning, and it makes predictions based on the following function ${ }^{55}$ :

$$
y(x)=\sum_{n=1}^{m} w_{n} K\left(x, x_{n}\right)+w_{0},
$$

where $w_{n}$ indicates the model weights and $K(.,$.$) is a kernel function. We applied two algorithms, SVM with$ linear kernel function and RVM with polynomial kernel function.

Cubist. Cubist, a rule-based regression tree algorithm, is based on the M5 theor $\mathrm{y}^{56}$. This model involves four main steps as follows: (1) growing a tree by branching data, (2) developing the model, (3) pruning the tree, and (4) smoothing the tree ${ }^{57}$.

Adaptive network-based fuzzy inference system (ANFIS). This model has been applied in different sciences. ANFIS works based on the fussy if/then rules $^{58}$ :

$$
\begin{aligned}
& \text { Rule } 1 \text { : if }\left(x \text { is } A_{1}\right) \text { and }\left(y \text { is } B_{1}\right) \text { then }\left(f_{1}=p_{1} x+q_{1} y+r_{1}\right) \\
& \text { Rule } 2 \text { : if }\left(x \text { is } A_{2}\right) \text { and }\left(y \text { is } B_{2}\right) \text { then }\left(f_{2}=p_{2} x+q_{2} y+r_{2}\right)
\end{aligned}
$$




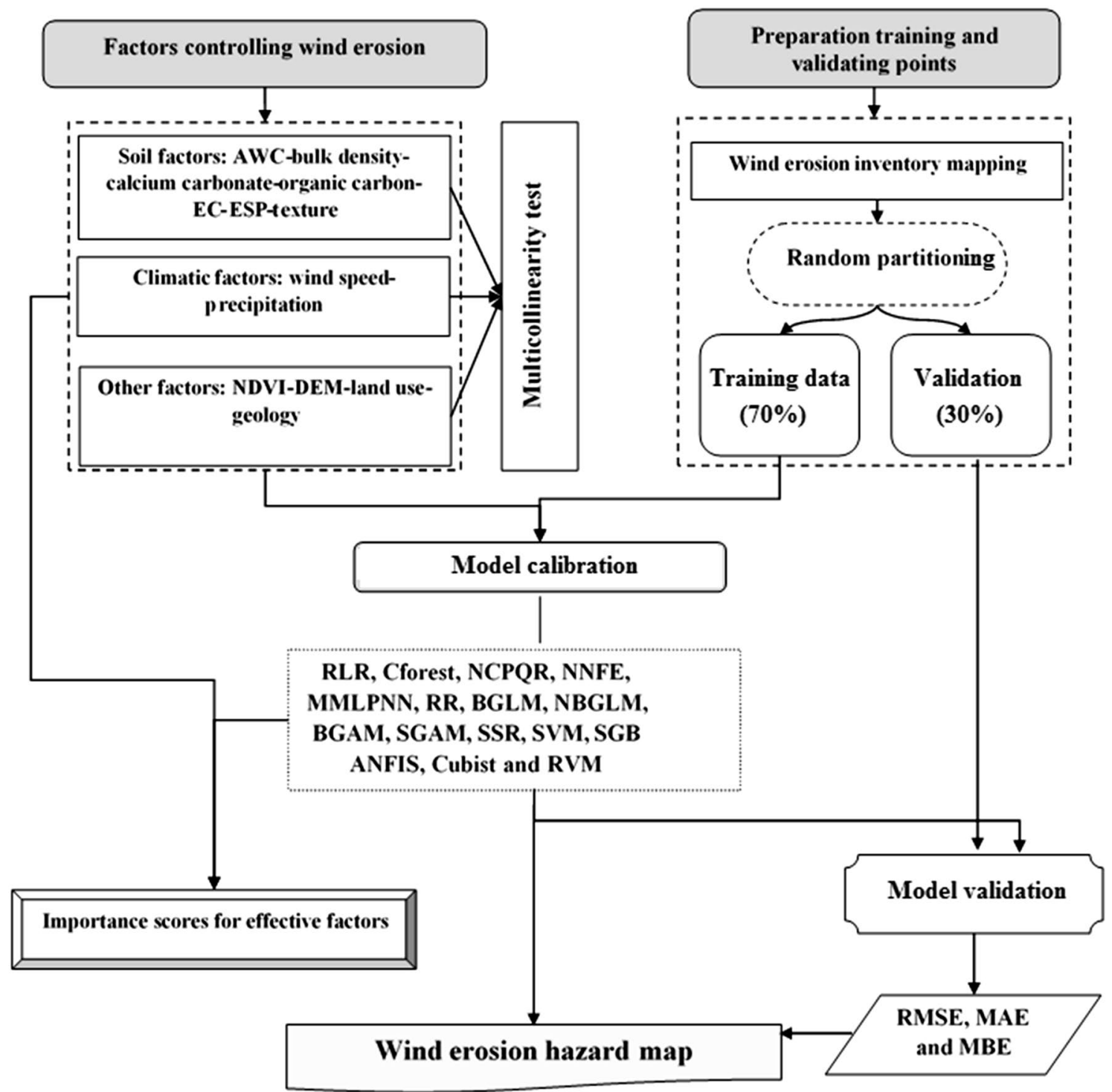

Figure 11. Flowchart of the methodology for mapping of wind erosion hazard.

where $\mathrm{x}$ and $\mathrm{y}$ are as input parameters for FIS, $\mathrm{f}$ as FIS output, $\mathrm{A}$ and $\mathrm{B}$ are fuzzy sets, and $\mathrm{p}, \mathrm{q}$, and $\mathrm{r}$ are parameters.

In all 16 models, the predicted values for pixels ranged between $0-1$. Therefore, we can divide susceptibility predictions into four classes (low (0-0.25), moderate (0.25-0.50), high (0.50-0.75) and very high (0.75-1)).

Assessment of model performance. In order to evaluate model performance in predicting land susceptibility to wind erosion hazard in the study area, three statistical methods comprising root mean square error (RMSE), mean absolute error (MAE) ${ }^{59,60}$ and mean bias error (MBE) were used:

$$
\begin{aligned}
R M S E & =\sqrt{\frac{\sum_{i=1}^{m}\left(v_{k}-v_{p}\right)^{2}}{m}} \\
M A E & =\frac{\sum_{i=1}^{m}\left|v_{k}-v_{p}\right|}{m} \\
M B E & =\frac{1}{m} \sum_{i=1}^{m}\left(v_{k}-v_{p}\right)
\end{aligned}
$$


where $m$ is number of the observations, $v_{k}$ and $v_{p}$ indicate the measured and predicted values, respectively. Also, a Taylor diagram was applied as a further test for assessing the performance of individual regression-based ML models $^{14}$.

Prioritization of the factors controlling wind erosion. Among the $16 \mathrm{ML}$ models tested, a model with the lowest error (RMSE, MAE, and MBE) was applied to quantify the relative importance of the factors controlling wind erosion. In this study, MMLPNN had the lowest error (with RMSE, MAE, and MBE $<0.002 \%$ ) and was therefore applied for determining the relative importance of the factors for wind erosion.

A brief overview of the main steps used in our methods is presented in Fig. 11.

Received: 6 January 2020; Accepted: 10 November 2020

Published online: 24 November 2020

\section{References}

1. Prospero, J. M., Ginoux, P., Torres, O., Nicholson, S. E. \& Gill, T. E. Environmental characterization of global sources of atmospheric soil dust identified with the Nimbus 7 Total Ozone Mapping Spectrometer (TOMS) absorbing aerosol product. Rev. Geophys. 40(1), $1-31$ (2002).

2. Goossens, D. On-site and off-site effects of wind erosion. In Wind Erosion on Agricultural Land in Europe (ed. Warren, A.) 29-38 (Luxembourg, European Commission, 2003).

3. Dahmardeh Behrooz, R., Gholami, H., Telfer, M. W., Jansen, J. D. \& Fathabadi, A. Uisng GLUE to pull apart the provenance of atmospheric dust. Aeolian Res. 37, 1-13 (2019).

4. Collins, A. L., Blackwell, M., Boeckx, P., Chivers, C. A., Emelko, M., Evrard, O., \& Harris, P. Sediment source fingerprinting: benchmarking recent outputs, remaining challenges and emerging themes. J. Soils Sedim. 1-34 (2020).

5. Rashki, A., Kaskaoutis, D. G., Goudie, A. S. \& Kahn, R. A. Dryness of ephemeral lakes and consequences for dust activity: The case of the Hamoun drainage basin, southeastern Iran. Sci. Total Environ. 463-464, 552-564 (2013).

6. Gholami, H., Rahimi, S., Fathabadi, A., Habibi, S., \& Collins, A. L. Mapping the spatial sources of atmospheric dust using GLUE and Monte Carlo simulation. Sci. Total Environ. 138090 (2020).

7. Schepanski, K., Tegen, I. \& Macke. A. Comparison of satellite based observations of Saharan dust source areas. Remote Sens. Environ. 123, 90-97 (2012).

8. Gholami, H., Mohamadifar, A. \& Collins, A. L. Spatial mapping of the provenance of storm dust: Application of data mining and ensemble modelling. Atmos. Res. 233(1), 104716 (2020).

9. Bondi, G., Creamer, R., Ferrari, A., Fenton, O. \& Wall, D. Using machine learning to predict soil bulk density on the basis of visual parameters: Tools for in-field and post-field evaluation. Geoderma 318, 137-147 (2018).

10. Pham, B. T. et al. A novel artificial intelligence approach based on multi-layer perceptron neural network and biogeography-based optimization for predicting coefficient of consolidation of soil. CATENA 173, 302-311 (2019).

11. Prasad, R., Deo, R. C., Li, Y. \& Maraseni, T. Soil moisture forecasting by a hybrid machine learning technique: ELM integrated with ensemble empirical mode decomposition. Geoderma 330, 136-161 (2018).

12. Gholami, H., Mohammadifar, A., Pourghasemi, H. R., \& Collins, A. L. A new integrated data mining model to map spatial variation in the susceptibility of land to act as a source of aeolian dust. Environ. Sci. Pollut. Res. 1-18 (2020).

13. Jha, S. K. \& Ahmad, Z. Soil microbial dynamics prediction using machine learning regression methods. Comput. Electron. Agric. 147, 158-165 (2018).

14. Gholami, H., Mohamadifar, A., Sorooshian, A. \& Jansen, J. D. Machine-learning algorithms for predicting land susceptibility to dust emissions: The case of the Jazmurian Basin, Iran. Atmos. Pollut. Res. 11, 1303-1315 (2020).

15. Pourghasemi, H. R., Yousefi, S., Kornejady, A. \& Cerda, A. Performance assessment of individual and ensemble data-mining techniques for gully erosion modeling. Sci. Total Environ. 609, 764-775 (2017).

16. Shao, Y. Physics and modelling of wind erosion. Atmos. Oceanogr. Sci. Library 37, 459 (2008).

17. Lang., B. Monotonic multi-layer perceptron networks as universal approximators. In International Conference on Artificial Neural Networks (ICANN), 31-37 (2005).

18. Gerling, L., Löschau, G., Wiedensohler, A. \& Weber, S. Statistical modelling of roadside and urban background ultrafine and accumulation mode particle number concentrations using generalized additive models. Sci. Total Environ. 134570 (2019).

19. Hagenauer, J., Omrani, H. \& Helbich, M. Assessing the performance of 38 machine learning models: The case of land consumption rates in Bavaria, Germany. Int. J. Geogr. Inf. Sci. 33(7), 1399-1419 (2019).

20. Keskin, H., Grunwald, S. \& Harris, W. G. Digital mapping of soil carbon fractions with machine learning. Geoderma 339, 40-58 (2019).

21. Chen, W. et al. A comparative study of logistic model tree, random forest, and classification and regression tree models for spatial prediction of landslide susceptibility. CATENA 151, 147-160 (2017)

22. Xu, Y. et al. Evaluation of machine learning techniques with multiple remote sensing datasets in estimating monthly concentrations of ground-level $\mathrm{PM}_{2.5}$. Environ. Pollut. 242, 1417-1426 (2018).

23. Sutton, C. D. Classification and regression trees, bagging, and boosting. Handb. Stat. (Elsevier) 24, 303-329 (2005).

24. Friedman, J. H. Greedy function approximation: A gradient boosting machine. Ann. Stat. 29(5), 1189-1232 (2001).

25. Friedman, J. H. Stochastic gradient boosting. Comput. Stat. Data Anal. 38, 367-378 (2002).

26. Moisen, G. G. Predicting tree species presence and basal area in Utah: A comparison of stochastic gradient boosting, generalized additive models, and tree-based methods. Ecol. Model. 199, 176-187 (2006).

27. Saadoud, D., Hassani, M., Peinado, F. J. M. \& Guettouche, M. S. Application of fuzzy logic approach for wind erosion hazard mapping in Laghouat region (Algeria) using remote sensing and GIS. Aeol. Res. 32, 24-34 (2018).

28. Chepil, W. S., Siddoway, F. H. \& Armbrust, D. V. Climate factor for estimating wind erodibility of farm fields. J. Soil Water Conserv. 17, 162-165 (1962).

29. Thornthwaite, C. W. An approach towards a rational classification of climate. Geogr. Rev. 38, $55-94$ (1948).

30. IUSS-WRB. World Reference Base for Soil Resources 2014, Update 2015. International Soil Classification System for Naming Soils and Creating Legends for Soil Maps. World Soil Resources Reports No. 106 (FAO, Rome, 2015).

31. Lamchin, M. et al. Assessment of land cover change and desertification using remote sensing technology in a local region of Mongolia. Adv. Space Res. 57, 64-77 (2016).

32. Bui, D. T., Pradhan, B., Lofman, O., Revhaug, I. \& Dick, O. B. Spatial prediction of landslide hazards in Vietnam: A comparative assessment of the efficacy of evidential belief functions and fuzzy logic models. CATENA 96, 28-40 (2012).

33. Huber, P. J. Robust Statistics (Wiley, New York, 1981).

34. Jackel, L. A. Estimating regression coefficients by minimizing the dispersion of the residuals. Ann. Math. Stat. 5, 1449-1458 (1972).

35. Siegel, A. F. Robust regression using repeated medians. Biometrika 69, 242-244 (1982). 
36. Rousseeuw, P. \& Yohai, V. Robust regression by means of S-estimators. Robust and non-linear time series. in (J. Franke, W. Hardle, R. D. Martin eds.) Lectures Notes in Statistics Vol. 26, 256-272 (Springer, New York, 1984).

37. Croux, C., Rousseeuw, P. J. \& Hossjer, O. Generalized S-estimators. J. Am. Stat. Assoc. 89, 1271-1281 (1994).

38. Yohai, V. J. High breakdown-point and high efficiency robust estimates for regression. Ann. Stat. 15, 642-656 (1987).

39. Breiman, l. Random forest. Mach. Learn. 45, 5-32 (2001).

40. Srivastava, R., Tiwari, A. N. \& Giri, V. K. Solar radiation forecasting using MARS, CART, M5, and random forest model: A case study for India. Heliyon 5(10), e02692 (2019).

41. Koenker, R. \& Bassett, G. Regression quantiles. Econometrica 46, 33-50 (1978).

42. Ma, H., Li, T., Zhu, H. \& Zhu, Z. Quantile regression for functional partially linear model in ultra-high dimensions. Comput. Stat. Data Anal. 129, 135-147 (2019).

43. Krasnopolsky, V.M. \& Chevallier, F. Some neural network applications in environmental sciences. Part II: Advancing computational efficiency of environmental numerical models. Neural Netw. 16, 335-348 (2003).

44. Heung, B. et al. An overview and comparison of machine-learning techniques for classification purposes in digital soil mapping. Geoderma 265, 62-77 (2016).

45. Horn, Z. C., Auret, L., McCoy, J. T., Aldrich, C. \& Herbst, B. M. Performance of convolutional neural networks for feature extraction in forth flotation sensing. IFAC-PapersOnLine 50(2), 13-18 (2017).

46. Canon, A.J. Multi-Layer Perception Neural Network with Optional Monotonicity Constraints. Package (2017).

47. Hoerl, A. E. \& Kennard, R. W. Ridge regression: Biased estimation for nonorthogonal problems. Technometrics 12(1), 50 (1970).

48. Saunders, C., Gammerman, A. \& Vovk, V. Ridge regression learning algorithm in Dual variables. in Proceeding ICML '98 Proceedings of the Fifteenth International Conference on Machine Learning, 515-521. San Francisco, CA, USA (1998).

49. Agostinelli, C., Valdora, M. \& Yohai, V. J. Initial robust estimation in generalized linear models. Comput. Stat. Data Anal. 134, 144-156 (2019).

50. Hosack, G. R., Hayes, K. R. \& Barry, S. C. Prior elicitation for Bayesian generalised linear models with application to risk control option assessment. Reliab. Eng. Syst. Saf. 167, 351-361 (2017).

51. Shirazi, M., Lord, D., Dhavala, S. S. \& Geedipally, S. R. A semiparametric negative binomial generalized linear model for modeling over-dispersed count data with a heavy tail: Characteristics and applications to crash data. Accid. Anal. Prevent. 91, 10-18 (2016).

52. Hastie, T. J. \& Tibshirani, R. J. Generalized additive models. Stat. Sci. 1(3), 297-310 (1986).

53. Ishwaran, H. \& Rao, J. S. Consistency of spike and slab regression. Stat. Probab. Lett. 81, 1920-1928 (2011).

54. Lempers, F. B. Posterior Probabilities of Alternative Linear Models (Rotterdam University Press, Rotterdam, 1971).

55. Tipping, E. The relevance vector machine. in NIPS Proceeding (2000).

56. Quinlan, R. Learning with continuous classes. in Proceedings of the 5th Australian Joint Conference on Artificial Intelligence, Hobart, Australia, 16-18 November 1992; 343-348 (1992).

57. Nguyen, H., Bui, X. N., Tran, Q. H. \& Mai, N. L. A new soft computing model for estimating and controlling blast-produced ground vibration based on hierarchical K-means clustering and cubist algorithms. Appl. Soft Comput. J. 77, 376-386 (2019).

58. Jang, J. S. R. ANFIS: Adaptive-network-based fuzzy inference system. IEEE Trans. Syst. 23(3), 665-685 (1993).

59. Gholami, H., Jafari TakhtiNajad, E., Collins, A. L. \& Fathabadi, A. Monte Carlo fingerprinting of the terrestrial sources of different particle size fractions of coastal sediment deposits using geochemical tracers: some lessons for the user community. Environ. Sci. Pollut. Res. 26, 23206 (2019).

60. Fan, M., Hu, J., Cao, R., Ruan, W. \& Wei, X. A review on experimental design for pollutants removal in water treatment with the aid of artificial intelligence. Chemosphere 200, 330-343 (2018).

\section{Acknowledgements}

The authors would like to thank the Faculty of Agriculture and Natural Resources, University of Hormozgan, Iran, for supporting this joint research project. Input by ALC was supported by the UKRI (UK Research and Innovation) Biotechnology and Biological Sciences Research Council (BBSRC) via grant award BBS/E/C/000I0330.

\section{Author contributions}

H.G. conceived the original idea of the research. Modelling work was undertaken by H.G. and A.M. H.G., D.T.B. and A.C. co-wrote the manuscript.

\section{Competing interests}

The authors declare no competing interests.

\section{Additional information}

Correspondence and requests for materials should be addressed to H.G. or D.T.B.

Reprints and permissions information is available at www.nature.com/reprints.

Publisher's note Springer Nature remains neutral with regard to jurisdictional claims in published maps and institutional affiliations.

(c) (i) Open Access This article is licensed under a Creative Commons Attribution 4.0 International cc. License, which permits use, sharing, adaptation, distribution and reproduction in any medium or format, as long as you give appropriate credit to the original author(s) and the source, provide a link to the Creative Commons licence, and indicate if changes were made. The images or other third party material in this article are included in the article's Creative Commons licence, unless indicated otherwise in a credit line to the material. If material is not included in the article's Creative Commons licence and your intended use is not permitted by statutory regulation or exceeds the permitted use, you will need to obtain permission directly from the copyright holder. To view a copy of this licence, visit http://creativecommons.org/licenses/by/4.0/.

(c) The Author(s) 2020 